\title{
La entrevista como herramienta para la evaluación de competencias genéricas: construcción de un consenso sobre evidencias
}

\author{
The interview as a tool for the assessment of generic \\ competences: building an evidence consensus \\ Manuel POBLETE RUIZ, Donna FERNÁNDEZ NOGUEIRA, Lucía CAMPO \\ CARRASCO y Marcelo NÖEL LÓPEZ \\ Universidad de Deusto
}

Recibido: Septiembre 2014

Evaluado: Diciembre 2014

Aceptado: Diciembre 2014

\section{Resumen}

La actual realidad educativa en la Educación Superior está caracterizada por la implementación del proceso de convergencia europeo. Un factor clave es el protagonismo del estudiante en el modelo de aprendizaje basado en competencias, donde el proceso de evaluación es un factor importante a desarrollar. En el presente estudio, cinco profesores de la Universidad de Deusto diseñaron una entrevista como herramienta de evaluación de cuatro competencias genéricas. La entrevista se aplicó a 21 estudiantes de un curso de emprendizaje social. Para la evaluación, se definieron lo que se considerarían evidencias de desempeño en las respuestas. Por último, se validaron los resultados aplicando el coeficiente de similitud de Gower. Los resultados sugieren una alta similitud en la evaluación de evidencias entre los cinco docentes, lo cual aporta fiabilidad a la medición. En conclusión, existiendo un consenso sobre los criterios de evaluación entre docentes, se puede mejorar la fiabilidad de las evaluaciones a los estudiantes y se aporta una herramienta para su evaluación.

Palabras clave: competencias genéricas, entrevista, evaluación, evidencia, consenso, educación superior.

\begin{abstract}
The current educational reality in the field of Higher Education is characterized by the implementation of the European process of convergence. A key factor is the student's leading role in the model of competence based learning, in which the process of evaluation is a key factor to be developed. In the current study, five university teachers from the University of Deusto designed an interview as a tool for the assessment of four generic competences. This interview was applied to 21 students in a social entrepreneurship course. In order to be consistent in the assessment, evidence of performance was previously defined. Finally, the Coefficient of Similarity of Gower was applied in the process of validation of results. These suggest a high similarity in the evaluation of evidences among the five teachers, thus providing
\end{abstract}


reliability to the measurement. In conclusion, the reliability of assessment can be improved by reaching a consensus in the evaluation criteria among professors and a tool for evaluation is provided in this paper.

Keywords: generic/transversal competences, interview, assessment-evaluation, evidences, consensus, higher education.

Una vez implantada la reforma del Espacio Europeo de Educación Superior (EEES), distintos estudios apuntan a que se han alcanzado algunas de las metas. Se han ajustado los cambios en los currículos de las titulaciones. Se ha mejorado la planificación de las asignaturas conforme a la filosofía ECTS (European Credit Transfer System). Se ha adoptado la conversión del “crédito” en la unidad básica de planificación, de implementación y de evaluación. No obstante, esas mismas investigaciones muestran también las metas por alcanzar en áreas como las metodologías, la evaluación, la tutoría o la formación del profesorado (Villa, Campo, Arranz, Villa y García, 2013; Rodríguez-Entrena y Rodríguez-Entrena, 2012; Raposo y González, 2010). En concreto, el aprendizaje por competencias está teniendo una mayor complejidad en su puesta en práctica que la prevista inicialmente. Las competencias genéricas se han significado como uno de los aspectos más novedosos e importantes del aprendizaje basado en competencias en el Espacio Europeo de Educación Superior. Sin embargo, una de las principales dificultades en el desarrollo del aprendizaje de las competencias genéricas es su evaluación.

La evaluación de competencias se ha demostrado como un momento crítico y clave dentro del proceso, cobrando mayor relieve en el momento en que se ha cambiado el objeto de evaluación, pasando de evaluar sólo conocimientos y en todo caso conocimientos y habilidades a evaluar una realidad sustancialmente diferente: el resultado de integrar conocimientos, habilidades, procedimientos, actitudes, valores. Si se quiere implantar este modelo de aprendizaje centrado en el estudiante, el profesor, además de planificar y organizar la docencia en función del nuevo sistema de créditos (ECTS), debe adoptar el rol de acompañante y guía del estudiante (Feixas, 2010; Tigelaar, Dolmans, Wolfhagen y van der Vleuten, 2004), pasando a ser una ayuda, un soporte, un medio para el desarrollo académico-profesional del estudiante, respetando y fomentando la autonomía de éste. Y esto parece que requiere y depende del desarrollo profesional del docente y de su formación. Asimismo, la innovación mediante nuevas técnicas y metodologías para las distintas fases del proceso de aprendizaje, pueden ayudar y apoyar al profesor en este reto al que se debe enfrentar.

El objetivo general de este estudio es analizar la utilidad de la entrevista como técnica de evaluación de competencias. Para el logro de este objetivo general se plantean los siguientes objetivos específicos:

- Medir la fiabilidad de la evaluación de competencias de la entrevista.

- Analizar la importancia del consenso entre los docentes que participan en la evaluación de competencias. 
- Considerar el proceso de consenso entre el profesorado implicado en la evaluación de las competencias como un espacio de formación continua.

- Definir niveles de evaluación de evidencias obtenidas de la entrevista.

En este estudio se presenta el diseño y aplicación de una entrevista de evaluación de competencias genéricas. A partir de esta aplicación, se muestra una técnica para el análisis y valoración de los datos obtenidos de la entrevista, basada en la definición de niveles de evidencia. Con una evaluación de evidencias entre jueces-evaluadores, se comprueba en un primer momento la fiabilidad de esta técnica de valoración mediante evidencias, obtenidas de la entrevista, para evaluar competencias genéricas. La entrevista puede aportar una mayor objetividad a la evaluación. De este modo, invirtiendo en una suficiente y necesaria capacitación del docente, se pueden lograr puntos de consenso en la valoración del desempeño del estudiante y dar una mayor fiabilidad a la evaluación.

Importancia del proceso de evaluación de competencias: la entrevista como un instrumento de evaluación de competencias.

Las competencias implican una combinación dinámica de conocimientos, comprensión, capacidades y habilidades (González y Wagenaar, 2006). Por ello, el proceso de evaluación en este nuevo marco requiere tener en cuenta no sólo una realidad que va más allá de conocimientos o conceptos. Al poseer las competencias genéricas altos contenidos de actitudes y valores resulta insuficiente hacer su evaluación mediante la aplicación de una prueba objetiva o una exposición teórica. En esta fase del proceso del aprendizaje el estudiante aprende, es evaluado y se da cuenta del recorrido que va realizando. Por ello, como afirman Baartman, Prins, Kirschner y Cees (2007), el docente debe evaluar los diferentes elementos o recursos (Tardif, 2006) de competencia y esta evaluación ha de realizarse de manera integrada.

Wiliam (2011) afirma que el proceso central en una formación efectiva es la evaluación, como medio para asegurarnos de que las actividades realizadas han alcanzado los resultados de aprendizaje que se pretendían. Si sobre la evaluación de las competencias hay un intenso debate, existe, no obstante, consenso acerca de que han de ser evaluadas a través del desempeño. Por ello la evaluación de competencias exige la definición de ciertos estándares con los cuales contrastar dicho desempeño. El objetivo principal de la evaluación no es la calificación, sino que el estudiante se dé cuenta de lo que ha aprendido, de lo que le falta por aprender, dónde encuentra dificultades y cómo salvarlas, etc. Como indica Gil-Flores "se pone el énfasis en la integración de la evaluación en el proceso de aprendizaje y en su carácter formativo, de modo que a través de un adecuado feedback la evaluación oriente de manera efectiva el aprendizaje de los estudiantes” ( 2012, p. 135).

Por otro lado, la entrevista es una técnica que ha sido utilizada en distintos ámbitos como herramienta válida para evaluar. Viene siendo utilizada en distintos ámbitos como la selección de personal en el ámbito laboral (Larson, 2002; Alcalá, 2001), en el ámbito de la psicología clínica (McConaughy, 2013; Perpiñá, 2012), o la entrevista periodística (Cantavella, 1996; Murphy y Dillon, 2010). La entrevista en el ámbito educativo se ha llevado a cabo, habitualmente para dialogar con los distintos agentes de 
la comunidad educativa (Zwiers y Morrissette, 1999). Asimismo, la entrevista es una técnica ampliamente aplicada en el mundo occidental, debido a que es un instrumento relativamente fácil de utilizar, permite recoger gran cantidad de información de los entrevistados y adaptarse a las características y necesidades de cada uno de ellos. Por lo tanto, es bien aceptada como instrumento de evaluación por entrevistados y entrevistadores.

Hay autores que han hablado de la importancia del diálogo en la educación (Freire, 1975). Para Freire el diálogo es el principal medio socializador y exhorta a todos a una conversión de la realidad educativa a través del diálogo, cuyo principal objetivo no es otro sino la auténtica comunicación entre las personas que les permite el encuentro, la liberación, donde prevalece la humildad para recibir lo que viene por parte del otro y para corregir nuestras equivocaciones, el respeto a sí mismos y hacia los demás.

La entrevista, en términos generales, es un diálogo entre dos o más personas: el entrevistador o entrevistadores que interrogan y el entrevistado o entrevistados que responden con el fin de tratar un asunto determinado. Se da en un entorno concreto y con una meta y condiciones determinadas. Es una técnica que, según el objetivo a lograr, puede tener distinta estructura, finalidades y modalidades.

Como prácticas que guardan relación con la entrevista se encuentran actividades desarrolladas en la antigüedad clásica, como es el método socrático, que utiliza las preguntas como instrumento principal. En ellas se basa la construcción de una buena entrevista. Las posibilidades del método socrático coinciden en cierta manera con el enfoque de la innovación docente actual, en el que el profesor no "enseña" al estudiante todo lo que tiene que saber, sino que es el estudiante el que construye el conocimiento, lo contrasta y evalúa, mediante el diálogo con el profesor (y otros agentes), que le ayudan a alcanzar por sí mismo el saber.

En este contexto educativo se crea la Entrevista de evaluación del aprendizaje focalizada en competencias, para ayudar a resolver en el proceso de evaluación un problema importante, haciendo que la evaluación sea una fase del proceso de aprendizaje, constituyendo un momento educador por excelencia.

El docente que evalúa e importancia del consenso sobre evidencias de desempeño del estudiante.

En el enfoque de la convergencia para la creación del Espacio Europeo de Educación Superior (EHEA. The European Higher Education Area, 1999), se enfatiza que hay que priorizar una pedagogía centrada en el aprendizaje frente a la pedagogía tradicional centrada en la enseñanza (Samuelowicz y Bain, 2001) y son también muchos los autores que coinciden en poner al docente como uno de los componentes determinantes para el cambio (Küster y Vila, 2012). El cambio exige, a este propósito, formación genérica para entender la nueva orientación del proceso educativo y formación específica en técnicas concretas, como puede ser la entrevista para la evaluación de competencias.

En relación a la entrevista de evaluación diseñada para este trabajo, se exige un contenido específico de formación sobre el rol que ha de asumir el profesor como 
entrevistador-evaluador. Por un lado, ha de dominar la técnica de la entrevista en general. Por otro lado, teniendo en cuenta que la evaluación de competencias encierra una complejidad por la realidad misma de las competencias y que las evidencias son las pruebas que aporta el estudiante para demostrar el desarrollo de una determinada competencia, en los elementos que la integran, corresponde al profesor la responsabilidad de diferenciar entre lo que es una evidencia de desempeño y lo que no lo es. La tarea es compleja, ya que el concepto de evidencia también lo es. Las evidencias no están preestablecidas, ya que son contextuales y plurales. Por tanto, no existen inventarios de las acciones que se convierten en evidencias para un determinado desempeño. Se hace necesaria en este proceso la definición consensuada del concepto de evidencia.

Si el profesor es parte de un equipo que va a llevar a cabo una evaluación de competencias, se impone la construcción de acuerdos acerca de los criterios a considerar sobre las evidencias. Klenowski (2011) señala que los docentes dan importancia a lograr acuerdos en la evaluación de las respuestas de los estudiantes. El presente trabajo, va más allá, subrayando la importancia de una construcción previa de significados, procedimientos y técnicas consensuadas para el proceso de evaluación. Este consenso previo facilitará el posterior ajuste y validación de las respuestas de los estudiantes.

Las evidencias son pruebas que se aportan y que no pueden sólo reducirse a lo observable, pudiendo ser directas o indirectas. Estas se construyen (se establecen a partir de una acción sobre el medio, siendo, por tanto, contextuales y plurales y no son neutrales ni perennes). La determinación de dichas evidencias forma parte de un proceso de construcción compartido entre los docentes implicados en el desarrollo de la competencia. Las evidencias son flexibles, no rígidas. En este trabajo y previo a la realización de las entrevistas, se diseñó un proceso de evaluación basado en los consensos sobre los criterios, indicadores y valoración de evidencias. No existiendo inventarios de las acciones que se convierten en evidencias para un determinado desempeño, se consideró la posibilidad de tomar como referencia la taxonomía de Bloom (1971).

\section{Método}

A partir de este enfoque sobre el proceso de evaluación de competencias y teniendo en cuenta la importancia del consenso entre los docentes evaluadores del desempeño del estudiante, se elaboró un instrumento de evaluación basado en la entrevista. Esta herramienta fue aplicada en el estudio piloto que se describe a continuación. Con los resultados de este estudio se pudo construir un análisis de evidencias inter-jueces (docentes evaluadores) para mostrar la fiabilidad de las evaluaciones.

Estudio piloto de la utilización de la Entrevista de Evaluación del aprendizaje focalizado en competencias.

Este trabajo se encuadra en el marco de un curso de postgrado de la Universidad de Deusto, realizado en el curso 2010-2011, financiado por la Diputación de Guipúzcoa, bajo el título de "Modelo de formación de emprendedores sociales basado en 
competencias”. El modelo de enseñanza-aprendizaje de este curso estaba diseñado en función de un enfoque competencial, contemplando competencias genéricas o transversales y competencias específicas, aunque para el estudio piloto sólo se contemplaron las competencias genéricas.

La fundamentación y adecuación de las competencias para el emprendedor social fue estudiada en su momento por el equipo coordinador del curso. Este modelo va en línea con estudios que sugieren la formación del emprendimiento social basado en competencias, como Miller, Wesley y Williams (2012). Así, las competencias genéricas clave seleccionadas para un emprendedor social, que se debían desarrollar y evaluar en el curso fueron: comunicación interpersonal, comportamiento ético, trabajo en equipo y liderazgo. Se eligieron las siguientes definiciones de competencia, niveles e indicadores, basados en el manual de Villa, Poblete y otros (2007) ${ }^{1}$ :

1. Comunicación interpersonal. Consiste en el establecimiento de una relación con otras personas a "través de la escucha empática" y la "expresión clara y asertiva de lo que se piensa y/o siente, por medios verbales y no verbales" (Villa, Poblete y otros, 2007: 237). Los indicadores respondían a dos niveles de dominio diferentes. La primera "emplea la escucha" del nivel 1 y "escucha y entiende las ideas de los demás aunque sean opuestas a las suyas, al nivel dos (Villa y Poblete, 2007: 238-239).

2. Comportamiento ético. Definido como la inclinación de forma positiva "hacia el bien moral de uno mismo o de los demás” (Villa y Poblete, 2007: 230). Incluye todo lo que significa la realización de la persona en busca de darle sentido a su vida, su compromiso hacia la justicia y el bien. Los indicadores que se evaluaron fueron dos del segundo nivel de dominio: "incorpora el sentido ético en otros ámbitos y se orienta en torno a él” y "mantiene coherencia entre lo que piensa y lo que hace” (Villa y Poblete, 2007: 231).

3. Trabajo en equipo. Es la forma activa de integrarse y colaborar en "la consecución de objetivos comunes con otras personas, áreas y organizaciones” (Villa y Poblete, 2007: 244). Los indicadores fueron en primer lugar, "realiza las tareas que le son asignadas dentro del grupo en los plazos requeridos", del nivel uno de dominio y del nivel dos, "se interesa por la importancia social de la actividad que se desarrolla en el grupo” (Villa y Poblete, 2007: 245-246).

4. Liderazgo. Definido como la influencia ejercida "sobre personas y/o grupos anticipándose al futuro y contribuyendo a su desarrollo personal y personal" (Villa y Poblete, 2007: 315) y "Anima al equipo a la consecución de objetivos".

Se asume en este estudio que se podría haber evaluado un tercer nivel de dominio de las competencias, que implicaría la influencia en el comportamiento de los demás. Se decidió centrar la evaluación en los dos primeros niveles ${ }^{2}$ de dominio.

\footnotetext{
${ }^{1}$ Este manual es el utilizado de manera institucionalizada para la evaluación en la Universidad de Deusto.

${ }^{2}$ Según Villa y Poblete (2007).
} 
Como primer paso en el desarrollo del instrumento, se acordaron las preguntas orientadas a obtener respuestas/evidencias sobre aspectos del desempeño de las competencias. Se acordaron las preguntas para poder evaluar la adquisición de las competencias. Teniendo en cuenta que se pretendía obtener evidencias del desempeño de la competencia, se decidió realizar dos tipos de preguntas. Las primeras se consideraban preguntas más de tipo conceptual o de comprensión y el segundo grupo ayudaba al evaluador a llegar al comportamiento real del entrevistado. La mayoría de las preguntas eran abiertas y algunas eran cerradas. La intención a la hora de seleccionar estas preguntas era que las respuestas fueran indicativas de los distintos elementos o recursos de la competencia (Tardif, 2006), así como poder identificar diferentes tipologías de evidencias.

Una de las dificultades a la hora de elaborar el protocolo, derivaba de la naturaleza de la competencia, en especial con la competencia de "comportamiento ético". Reflexionar sobre los actos de cada uno es fundamental, pero algunos ven en ello cierta subjetividad. Sin embargo, como menciona González-Bernal y otros (2007) esta reflexión tiene que "convertirse en acción” y los resultados de esta acción, sí son evaluables. Bolívar (2005) define la competencia ética no sólo en el ámbito personal sino sobre todo en el profesional. Asimismo, la clasificación de competencias de Sáenz y López (en prensa) muestra la relevancia y contribución del comportamiento ético en el perfil del emprendedor social.

A pesar de la dificultad en diseñar preguntas para evaluar la competencia de comportamiento ético, se creyó conveniente, tras la justificación teórica, integrar esta competencia en el instrumento de evaluación. El protocolo final elaborado, con una muestra de preguntas realizadas, fue el siguiente:

\begin{tabular}{|c|l|l|}
\hline \multirow{2}{*}{ COMPETENCIA } & $\begin{array}{c}\text { INDICADOR DE } \\
\text { EVALUACIÓN }\end{array}$ & \multicolumn{1}{|c|}{$\begin{array}{c}\text { EJEMPLO DE PREGUNTA DE LA } \\
\text { ENTREVISTA }\end{array}$} \\
\hline Comunicación & Emplea la escucha. & $\begin{array}{l}\text { ¿Qué es para ti la escucha en comunicación } \\
\text { interpersonal? } \\
\text { Cuéntame alguna situación en que la hayas } \\
\text { practicado. ¿Qué has hecho? }\end{array}$ \\
\cline { 2 - 4 } & $\begin{array}{l}\text { Escucha y entiende las } \\
\text { ideas de los demás } \\
\text { aunque sean opuestas a } \\
\text { las suyas. }\end{array}$ & $\begin{array}{l}\text { ¿Recuerdas alguna situación en la que no } \\
\text { hayas estado de acuerdo con alguien? } \\
\text { ¿Cuáles eran los puntos de vista que se } \\
\text { defendían? } \\
\text { ¿Cuál era el punto de vista más opuesto al } \\
\text { tuyo? } \\
\text { ¿Qué parte de razón tenía? }\end{array}$ \\
\hline $\begin{array}{c}\text { Comportamiento } \\
\text { ético }\end{array}$ & $\begin{array}{l}\text { Incorpora el sentido } \\
\text { ético en otros ámbitos } \\
\text { y se orienta en torno a } \\
\text { él. }\end{array}$ & $\begin{array}{l}\text { ¿ué es para ti un comportamiento ético? } \\
\text { Cuéne alguna situación en que lo hayas } \\
\text { ¿Te definirías como una persona ética? Da } \\
\text { razones y explica algún momento de tu vida } \\
\text { que dé valor a tu respuesta. }\end{array}$ \\
\hline
\end{tabular}




\begin{tabular}{|c|c|c|}
\hline & $\begin{array}{l}\text { Mantiene coherencia } \\
\text { entre lo que piensa y lo } \\
\text { que hace. }\end{array}$ & $\begin{array}{l}\text { ¿Recuerdas alguna situación en que se } \\
\text { demuestre tu coherencia entre lo que piensas y } \\
\text { lo que haces? ¿Puedes describirla? } \\
\text { ¿Con tu grupo de amigos has hecho algo con } \\
\text { lo que no estás de acuerdo? } \\
\text { Explica la situación y las razones de tu } \\
\text { decisión. } \\
\text { Recientemente ¿has tenido que ofrecer ayuda a } \\
\text { alguien fuera de tu grupo social? ¿Cuándo? } \\
\text { ¿Por qué? ¿Cómo has reaccionado? ¿Qué has } \\
\text { sentido? }\end{array}$ \\
\hline \multirow[t]{2}{*}{ Trabajo en equipo } & $\begin{array}{l}\text { Realiza las tareas que } \\
\text { le son asignadas dentro } \\
\text { del grupo en los plazos } \\
\text { requeridos. }\end{array}$ & $\begin{array}{l}\text { ¿Cuál ha sido la última vez que has trabajado } \\
\text { en equipo? ¿Qué te ha correspondido hacer? } \\
\text { ¿Cómo te las arreglaste? } \\
\text { ¿Entregaste tu parte en el tiempo exigido? } \\
\text { ¿Por qué? }\end{array}$ \\
\hline & $\begin{array}{l}\text { Se interesa por la } \\
\text { importancia social de } \\
\text { la actividad que se } \\
\text { desarrolla en el grupo. }\end{array}$ & $\begin{array}{l}\text { ¿Qué repercusión tuvo ese trabajo hacia fuera } \\
\text { del grupo? }\end{array}$ \\
\hline Liderazgo & $\begin{array}{l}\text { Anima al equipo a la } \\
\text { consecución de } \\
\text { objetivos. }\end{array}$ & $\begin{array}{l}\text { ¿Influyes en que los demás trabajen por lograr } \\
\text { el objetivo grupal? ¿Cómo? } \\
\text { ¿Animas a tus compañeros para que presenten } \\
\text { ideas que faciliten la consecución del objetivo } \\
\text { del grupo? } \\
\text { ¿Reconoces las buenas ideas de tus } \\
\text { compañeros cuando las presentan? } \\
\text { ¿Cómo crees que tu actuación ha influido en el } \\
\text { desempeño del grupo? }\end{array}$ \\
\hline
\end{tabular}

Tabla 1. Ejemplo de protocolo de entrevista de evaluación de competencias genéricas. Fuente: elaboración propia.

A partir del protocolo de la Tabla 1, se llevaron a cabo las entrevistas de evaluación. La finalidad de estas entrevistas era obtener los datos que mostrasen si un estudiante había alcanzado ese nivel de competencia y si su desempeño mostraba estas competencias genéricas en el marco del curso impartido. Asimismo, se construyeron unos niveles de evidencias de desempeño basadas en el consenso inter-jueces.

\section{Acuerdo sobre los niveles o grados de evidencia}

Se describe a continuación el procedimiento mediante el cual se definieron los niveles correspondientes y el proceso de evaluación realizado.

El gran reto de este trabajo fue la medición de los datos obtenidos de la entrevista o niveles de evidencia demostrada en las respuestas de los estudiantes entrevistados. Así, con la búsqueda y estudio de la bibliografía en este ámbito, se llegó a un modelo 
adaptado a la presente investigación. Este proceso se basó en la categorización de comportamientos y metas educativas de Benjamín Bloom (1971). Es necesario realizar una evaluación formativa que contribuya a la regulación de los aprendizajes. Sólo conociendo el nivel de dominio alcanzado por el estudiante en una competencia será posible orientar el aprendizaje futuro. Tomar como referencia esta taxonomía, permitió a los evaluadores trabajar con niveles y definición de niveles de respuesta, así como adoptar un criterio compartido de valoración de las respuestas.

El equipo de evaluadores, realizadores de este estudio, basándose en estas etapas de aprendizaje de Bloom y otros (1973), definió cinco niveles, con los que se medirían las respuestas obtenidas de los entrevistados. Se muestra a continuación una descripción de los niveles de evidencia, analizados desde los principales elementos de la competencia (conocimiento, actitud, comportamiento) y se añaden algunos ejemplos. Estos niveles se plantean desde un carácter integrador, donde cada nivel supone el logro de los anteriores:

1. La conceptualización.

- Conocimiento: Hay una conceptualización básica y una cierta preocupación, un pensamiento, algún grado de conciencia en esa competencia.

- Actitud: No se manifiesta aún una actitud clara hacia esta competencia.

- Comportamiento: no se muestra desempeño de la competencia a un nivel de ejecución.

- Ejemplo, "a mí me gustaría tener más tiempo para preparar algunas reuniones. A veces el día a día te come... me gustaría tener alguna herramienta más y sobre todo tiempo, pero si alguna herramienta más para dinamizar o plantear cosas”.

2. La reflexión.

- Conocimiento: Muestra comprensión del concepto.

- Actitud: Manifiesta en su discurso una actitud positiva hacia la competencia.

- Comportamiento: No se muestra ejecución de la competencia.

- Ejemplo, "para dirigir, hay que escuchar a los compañeros"; "hay que tener unos principios para andar por la vida".

3. La toma de postura.

- Conocimiento: Muestra comprensión del concepto.

- Actitud: Existe un posicionamiento positivo hacia la incorporación de la competencia en su propio desempeño.

- Comportamiento: No indica la ejecución de la competencia

- Ejemplo, "soy partidario de que todos hablen y participen"; "yo me posiciono siempre de parte del más débil”.

4. La actuación.

- Conocimiento: Muestra comprensión del concepto.

- Actitud: Existe un posicionamiento positivo hacia la incorporación de la competencia en su propio desempeño. 
- Comportamiento: Es el paso de la intención a la actuación, hay una ejecución clara.

- Ejemplo, "trato de...” sería una simple intención, pero pasaría a de nivel cuatro cuando se muestra ejecución. Por ejemplo, sobre el indicador de liderazgo: "cómo hacemos, no hacemos y tal... (...) Entonces yo, al intentar poner esas dos independientemente y luego como la unión de las dos hace que se solucionen los problemas. ¿eso les dijiste? Sí, y eso es lo que ha tirado para adelante. (...) Sé ver las capacidades de las demás, 'qué idea más buena tienes' 'eso estupendo', me sale. No soy muy buena en ideas, pero unir lo que ha dicho una con la otra...eso se me da mejor. (...) El viernes (grupo proyecto), me acuerdo de 'Qué idea más buena’ pero lo que no me acuerdo es cuál era la idea”.

5. La integración.

- Conocimiento: Muestra comprensión del concepto

- Actitud: Existe un posicionamiento positivo hacia la incorporación de la competencia en su propio desempeño. Realiza un juicio crítico sobre su comportamiento y se responsabiliza del mismo.

- Comportamiento: Existe un desempeño de la competencia. Requiere que el sujeto integre en su explicación la razón que le ha llevado a una actuación concreta.

- Un ejemplo de una evidencia de la escucha es la siguiente: "Noté que estaba mal, fuimos a tomar un café y le pregunté si le pasaba algo. Me callé y le dejé hablar. Repetí en pocas palabras lo que le había entendido y le hice alguna pregunta..."

Con los datos de la aplicación de las entrevistas, así como con la definición de los niveles de evidencia, se procedió al análisis de las respuestas obtenidas en las entrevistas. La metodología y procedimiento de la aplicación experimental se describen a continuación.

En la fase experimental de este estudio se utilizó como instrumento la entrevista semiestructurada organizada en cuatro partes, una para cada competencia genérica a evaluar (Comunicación, Comportamiento ético, Trabajo en equipo y Liderazgo). La finalidad de esta entrevista era evaluar las competencias genéricas de una muestra de estudiantes.

\section{Participantes}

Los participantes de este estudio fueron 21 estudiantes del curso "Modelo de emprendedores sociales basado en competencias" impartido por la Universidad de Deusto. En la programación del Curso se asignó una duración de 150 horas administradas durante un semestre académico. Todos los estudiantes trabajaban en el ámbito social y acudían al curso de manera voluntaria para especializarse en ciertas 
competencias específicas y genéricas de su ámbito laboral. La entrevista era uno de los instrumentos utilizados para evaluar las cuatro competencias genéricas propuestas en el curso. Existía también un informe de autoevaluación y una evaluación grupal.

El procedimiento para la realización de cada entrevista, con una duración en torno a los cuarenta y cinco minutos se desarrolló según los siguientes pasos. En primer lugar se explicó a cada participante el objetivo de esta investigación. Los estudiantes aceptaron que la entrevista fuera grabada, por lo que se obtuvo una muestra válida de 21 entrevistas. Estas entrevistas fueron realizadas, aunque por entrevistadores/evaluadores diferentes, con el mismo protocolo sobre las cuatro competencias genéricas mencionadas (Comunicación, Comportamiento ético, Trabajo en equipo y Liderazgo).

En segundo lugar los cinco evaluadores, tuvieron una formación en grupo con el fin de asegurar el mismo enfoque y la aplicación de un mismo procedimiento. Todos conocían el protocolo, la finalidad de las preguntas y la valoración de las respuestas mediante los niveles de evidencia consensuados. Como tercer paso se procedió a realizar las entrevistas, que se llevaron a cabo en las instalaciones de la Universidad de Deusto, en el mes de mayo de 2011, al finalizar el desarrollo del curso de "Modelo de emprendedores sociales basado en competencias”.

\section{Fases para el análisis de los resultados de las entrevistas}

Fase 1: Selección de evidencias y determinación de grados o niveles.

Cada evaluador valoró todas las evidencias recopiladas, siguiendo los niveles o grados definidos y consensuados:

1. La conceptualización

2. La reflexión

3. La toma de postura

4. La actuación

5. La integración

Una vez realizadas, las entrevistas fueron transcritas por cada uno de los evaluadores, que tenían como contraste la grabación correspondiente. Cada evaluador/a (que cumplió el rol de entrevistador/a) eligió lo que él/ella consideraba que era una evidencia de que el entrevistado/a desempeñaba o no una determinada competencia. La evaluación de las evidencias se realizó de manera individual, para integrar después estos datos en una base de datos común. 
Fase 2: Evaluación inter-jueces.

Construida la base de datos común de evaluación de evidencias, todos los evaluadores adoptaron un nuevo rol, el de jueces de las valoraciones de las evidencias de los demás. Esto se realizó con el fin contrastar las evaluaciones atribuidas por los diferentes evaluadores. La Tabla 2 muestra el número de evidencias recogidas por cada evaluador, así como el número total de las evidencias a analizar.

\begin{tabular}{cccccc|}
$\begin{array}{c}\text { Evaluador } \\
\mathbf{1}\end{array}$ & $\begin{array}{c}\text { Evaluador } \\
\mathbf{2}\end{array}$ & $\begin{array}{c}\text { Evaluador } \\
\mathbf{3}\end{array}$ & $\begin{array}{c}\text { Evaluador } \\
\mathbf{4}\end{array}$ & $\begin{array}{c}\text { Evaluador } \\
\mathbf{5}\end{array}$ & Total \\
\hline 4 & 5 & 4 & 4 & 4 & 21 \\
\hline 37 & 23 & 16 & 34 & 34 & 144 \\
\hline
\end{tabular}

Tabla 2. Entrevistas realizadas y evidencias aportadas por cada evaluador/a.

En la Tabla 3 se pueden observar estas evidencias clasificadas según los indicadores correspondientes a las cuatro competencias evaluadas en la entrevista:

\begin{tabular}{|c|c|l|c|}
\hline \multirow{2}{*}{ COMPETENCIA } & $\begin{array}{c}\text { Número } \\
\text { de } \\
\text { indicador }\end{array}$ & \multicolumn{1}{|c|}{ Indicador de evaluación } & $\begin{array}{c}\text { Número de } \\
\text { Evidencias }\end{array}$ \\
\hline \multirow{2}{*}{ COMUNICACIÓN } & 1 & Emplea la escucha & 21 \\
\cline { 2 - 4 } COMPORTAMIENTO & 2 & $\begin{array}{l}\text { Escucha y entiende las ideas de los demás } \\
\text { aunque sean opuestas a las suyas. }\end{array}$ & 21 \\
\cline { 2 - 4 } ÉTICO & 3 & $\begin{array}{l}\text { Incorpora el sentido ético en otros ámbitos y } \\
\text { se orienta en torno a él. }\end{array}$ & 21 \\
\hline \multirow{2}{*}{\begin{tabular}{c} 
TRABAJO EN EQUIPO \\
\cline { 2 - 4 }
\end{tabular}} & 5 & $\begin{array}{l}\text { Mantiene coherencia entre lo que piensa y lo } \\
\text { que hace. }\end{array}$ & 20 \\
\hline LIDERAZGO & 7 & $\begin{array}{l}\text { Realiza las tareas que le son asignadas } \\
\text { dentro del grupo en los plazos requeridos. }\end{array}$ & $\begin{array}{l}\text { Se interesa por la importancia social de la } \\
\text { actividad que se desarrolla en el grupo. }\end{array}$ \\
\hline
\end{tabular}

Tabla 3. Evidencias extraídas según las competencias e indicadores evaluados.

Es importante señalar que se acordó incorporar a los cinco niveles inicialmente previstos un nivel cero, que incluiría las evidencias que, a pesar de haber sido elegidas por un evaluador para estar en el listado, no se consideraban evidencia del desempeño por parte de otro de los jueces. 
Con el objetivo de calcular el acuerdo interno inter-jueces de las evaluaciones, se utilizó el coeficiente de similitud de Gower (1971), que hace referencia a la medición y la analogía entre los resultados aportados por individuos en base a tipos de información distintos, con la posibilidad de que existan vacíos en ella (Cf. Zegers, 1991).

Para obtener el coeficiente de Gower, se dispusieron las evaluaciones de las evidencias según los cinco evaluadores y se compararon dos a dos. De este modo, se obtuvo un coeficiente en un rango de 0 (no existe acuerdo) a 1 (acuerdo total entre los evaluadores). Se considera que entre 0.65 y 0.80 se alcanza un nivel aceptable de acuerdo entre los evaluadores. Los coeficientes de Gower mayores de 0.80 representan un alto acuerdo entre evaluadores y los menores de 0.65 un bajo nivel de acuerdo (Bakker et al., 2011, p. 128).

\section{Resultados}

Los resultados obtenidos para mostrar la utilidad de la herramienta desarrollada y aplicada para evaluar las competencias genéricas mediante la entrevista, contando con que se obtuvieron 144 evidencias seleccionadas para el estudio, en las cuales se evaluaban cuatro competencias genéricas de 21 participantes, se presentan en la Tabla 4.

\begin{tabular}{lcccc}
\hline & Evaluador 2 & Evaluador 3 & Evaluador 4 & Evaluador 5 \\
\hline Evaluador 1 & 0,78 & 0,81 & 0,77 & 0,82 \\
\hline Evaluador 2 & & 0,88 & 0,81 & 0,85 \\
\hline Evaluador 3 & & & 0,90 & 0,91 \\
\hline Evaluador 4 & & & & 0,85 \\
\hline
\end{tabular}

Tabla 4. Resultados del coeficiente de similitud de Gower de la evaluación inter-jueces.

Como se puede observar, todos los coeficientes (excepto dos) son superiores a .80 . Aparecen dos coeficientes (entre el evaluador $1 \mathrm{y}$ evaluador 2, de .78 y, entre el evaluador $1 \mathrm{y}$ el evaluador 4 , de .77, que son inferiores a .80, pero que indican aun así un grado muy aceptable de acuerdo inter-jueces.

Asimismo, se realizó un análisis de este coeficiente según los siete indicadores de evaluación de la entrevista, con el fin de observar a nivel más localizado si existe acuerdo inter-jueces (ver tablas en el Anexo 1. Resultados del Coeficiente de Gower en los 7 indicadores de las cuatro competencias evaluadas).

En lo que respecta a la competencia de Comunicación, encontramos una confiabilidad alta entre los evaluadores 2, 3, 4 y 5. Sin embargo, podemos observar que el evaluador 1 tiene valores bajos, incluso menores de .65 (de .61 a .63) con tres de los otros evaluadores. Estas puntuaciones más bajas no se repiten en ningún otro indicador y tanto el evaluador 1 como los otros cuatro, obtienen coeficientes considerados como niveles aceptables de acuerdo, de hecho más altos de .70, así como de alto acuerdo (mayores de .80). Los mayores acuerdos entre los evaluadores se han encontrado en los indicadores de las competencias de trabajo en equipo y liderazgo (indicadores 5, 6 y 7), 
donde prácticamente la totalidad de los evaluadores han obtenido coeficientes superiores a .80. Encontramos algunos valores cercanos a 1 en estos indicadores, lo que significaría un acuerdo total entre los jueces.

\section{Discusión}

El objetivo de este estudio era analizar la utilidad de la entrevista como técnica para la evaluación de competencias. Para ello, se diseñó un modelo de entrevista, así como un procedimiento consensuado de medición de evidencias de las respuestas. Los resultados más relevantes indicaron que se logró una clara similitud en las respuestas de los evaluadores a la hora de valorar las evidencias sobre competencias genéricas. Puede deducirse, por tanto, que los evaluadores, tras una formación sobre el procedimiento para la medición de evidencias, han evaluado de manera similar. Por ello, puede afirmarse que, a partir de esta entrevista de evaluación de competencias genéricas, se evalúan las evidencias obtenidas y se valora el desempeño del estudiante en estas competencias.

Por otro lado, cabe destacar la importancia de la formación de los docentes evaluadores, así como de la construcción de consenso entre los mismos, como factor que incide de manera clara en los resultados. Así, creemos que es importante esta coordinación entre los docentes con el fin de alcanzar unos criterios comunes que aporten transparencia, oportunidad y fiabilidad a la evaluación. Este trabajo va así en concordancia con un enfoque de la docencia en el que "la práctica reflexiva es la base de la profesionalidad efectiva" (Biggs, 2006) y esto incluye la enseñanza universitaria (Brockbank y McGill, 1998). Esto supone un reto para las instituciones, donde los tiempos para la docencia se ven sobrecargados.

Otra limitación que se puede encontrar en el presente estudio, es que se centra en una evaluación final basada en una herramienta como es la entrevista. A pesar de que a partir de los resultados se puede afirmar que este procedimiento aporta una manera eficaz de evaluación de competencias, recordamos que la evaluación centrada en el estudiante es más compleja. Se necesita evaluar distintos elementos y niveles del aprendizaje, que deben ser medidos desde diferentes perspectivas y, por tanto, con varios instrumentos. Por ello, se recomienda la triangulación de herramientas, así como la evaluación enfocada en el proceso de aprendizaje como elementos básicos para una evaluación basada en competencias. De hecho, la entrevista no debería ser el único instrumento de evaluación, entre otras razones, por la posibilidad de sesgo en el discurso del estudiante.

En resumen, este estudio aporta una herramienta para la evaluación de competencias genéricas y una propuesta para la definición de niveles de evidencias que ayuden al docente a ubicar los resultados de aprendizaje de sus estudiantes. Como apuntan Asikainen, Parpala, Virtanen y Lindblom-Ylänne (2013, p. 215) “evaluar distintos niveles de comprensión es más apropiado para la evaluación de resultados de aprendizaje”. 


\section{Conclusiones}

La entrevista como herramienta de evaluación de competencias se ha revelado en este estudio como instrumento efectivo para evaluar competencias, uno de los retos del nuevo enfoque en el proceso de aprendizaje propuesto por el Espacio Europeo de Educación Superior (EEES). Los desafíos y los retos relacionados con la investigación en evaluación de las competencias genéricas, están relacionados con una forma de aprendizaje holístico, integral y equilibrado, entre el profesorado por una parte y el estudiante por la otra, como participante activo en su proceso de formación.

Este trabajo demuestra que la variabilidad derivada de las diferentes formas de comprender las competencias y las evidencias, así como de evaluar los niveles competenciales, se ve reducida en buena medida al construir definiciones y niveles consensuados entre el equipo de profesores que participan en dicha evaluación. Conviene recordar que este es uno de los problemas más importantes a que se enfrenta el profesorado al evaluar las competencias. Por lo que es esencial encontrar formas para reducir la variabilidad y los sesgos naturales permitiendo evaluaciones más fiables y objetivas.

Al mismo tiempo, esta interacción y diálogo pueden ser comprendidos como una auténtica oportunidad de aprendizaje y formación continua no formal del profesorado, vinculado a los propios procesos de trabajo. Esto beneficiaría, por un lado, el clima de trabajo existente en el equipo de profesorado que imparte asignaturas afines o que sean de un mismo departamento. Por otro lado, a nivel individual, aumentaría la confianza del profesor en el sistema de evaluación utilizado y en el convencimiento de que el estudiante comprenderá y aceptará este modelo de evaluación de competencias. No cabe duda que todo ello tendería a fomentar prácticas de mayor calidad, aspecto central para el aprendizaje del estudiante.

Por último, parece razonable afirmar que el proceso que los equipos de profesores deberían llevar a cabo será paralelo al que se ha seguido por el equipo de esta investigación. De cara al futuro, se sugiere que en sucesivas investigaciones se continúe profundizando en este tema dado la escasez de publicaciones en este ámbito y frente a la necesidad que tiene el docente de contar con técnicas e instrumentos fiables y objetivos para la evaluación de competencias.

\section{Referencias bibliográficas}

ALCALÁ, M. A. (Ed.). (2001). La entrevista de selección. Manual para el entrevistador y para el entrevistado. Madrid: CIE. Dossat 2000 Management.

ASIKAINEN, H., PARPALA, A., VIRTANEN, V. y LINDBLOM-YLÄNNE, S. (2013). The relationship between student learning process, study success and the nature of assessment: A qualitative study. Studies in Educational Evaluation, 39(4), 211-217. doi:10.1016/j.stueduc.2013.10.008 
BAARTMAN, L. K. J., PRINS, F., KIRSCHNER, P. A., y VAN DER VLEUTEN, C. P. M. (2007). Determining the quality of competence assessment programs: a selfevaluation procedure. Studies in Educational Evaluation, 33(3-4), 258-281. doi:10.1016/j.stueduc.2007.07.004

BAKKER, M. E. J., ROELOFS, E. C., BEIJAARD, D., SANDERS, P. F., TIGELAAR, D. E. H., y VERLOOP, N. (2011). Video portfolios: The development and usefulness of a teacher assessment procedure. Studies in Educational Evaluation, 37(2-3), 123-133. doi:10.1016/j.stueduc.2011.04.007

BIGGS, J. (2006). Calidad del aprendizaje universitario. Madrid: Narcea.

BLOOM, B. S., Engelhart, M. D., Furst, E. J., Hill, W., y Krathwohl, D. (1971). Taxonomía de los objetivos de la educación (2 ${ }^{\circ}$ edición). Tomo I: Ámbito del conocimiento. Alcoy: Marfil S.A.

BLOOM, B. S., Masia, B. B., y Krathwohl, D. R. (1973). Taxonomía de los objetivos de la educación ( $2^{\circ}$ edición). Tomo II: Ámbito de la afectividad. Alcoy: Marfil S.A.

BOLÍVAR, A. (2005). El lugar de la ética profesional en la formación universitaria. Red Revista Mexicana de Investigación Educativa., 10(24), 93-123.

BROCKBANK, A. y McGILL, I. (1998). Facilitating Reflective Learning in Higher Education. Buckingham: The Society of Research into Higher Education and Open University Press.

CANTAVELLA, J. (1996). Manual de la Entrevista Periodística. Barcelona: Ariel.

EHEA. The European Higher Education Area. (1999). The Bologna Declaration of 19th June 1999: Joint declaration of the European Ministers of Education. Bologna: EHEA. Retrieved from http://www.eees.es/pdf/Declaracion_Bolonia.pdf

FEIXAS, M. (2010). Enfoques y concepciones docentes en la universidad. Relieve: Revista ELectrónica de Investigación y EValuación Educativa, 16(2), 1-27.

FREIRE, P. (1975). Pedagogía del oprimido. Madrid: Siglo XXI.

GIL-FLORES, J. (2012). La evaluación del aprendizaje en la universidad según la experiencia de los estudiantes. Estudios Sobre Educacion, 22, 133-153.

GONZÁLEZ, J., \& WAGENAAR, R. (2003). Tuning Educational Structures in Europe. Informe Final: Fase 1. Bilbao: Universidad de Deusto.

GONZÁLEZ-BERNAL, M. R., GÓMEZ, L. S., ESPINOSA, J. C., Cárdenas Díaz, D. C., Garzón Yepes, Y., Montoya Rivas, E. M., Tarquino Prieto, J. E. (2007). Evaluation by competences of the ethical dimension in the formation of psychologists in Colombia. Diversitas: Perspectivas En Psicología, 3(1), 11-23.

GOWER, J. C. (1971). A general coefficient of similarity and some of its properties. Biometrics, 27, 857-74. 
KLENOWSKI, V. (2011). Assessment for learning in the accountability era: Queensland, Australia. Studies in Educational Evaluation, 37(1), 78-83. doi:10.1016/j.stueduc.2011.03.003

KÜSTER, I., y VILA, N. (2012). El docente universitario y sus efectos en el estudiante. Estudios Sobre Educación, 23, 157-182.

LARSON, W. (2002). Realizar entrevistas de trabajo. Madrid: Prentice Hall.

MCCONAUGHY, S. H. (2013). Clinical Interviews for Children and Adolescents, Second Edition: Assessment to Intervention (Second Edition, Lay-Flat edition.). New York: The Guilford Press.

MILLER, T. L., WESLEY, C. L., y WILLIAMS, D. E. (2012). Educating the Minds of Caring Hearts: Comparing the Views of Practitioners and Educators on the Importance of Social Entrepreneurship Competencies. Academy of Management Learning \& Education, 11(3), 349-370.

MURPHY, B. C., y DILLON, C. (2010). Interviewing in Action in a Multicultural World (4 edition.). Australia: United States: Cengage Learning.

PERPIÑÁ, C. (Ed.). (2012). Manual de la entrevista psicológica. Madrid: Pirámide.

RAPOSO, M., y GONZÁLEZ, M. (2010). La creación del EEES desde el punto de vista de equipos directivos y profesorado. Enseñanza y Teaching, 28, 79-95.

RODRÍGUEZ-ENTRENA， M., y RODRÍGUEZ-ENTRENA， M. J. (2012). Preferencias educativas de los estudiantes postgraduados ante los cambios del Espacio Europeo de Educación Superior. Estudios Sobre Educación, 23, 183-203.

SÁENZ BILBAO, N. y LÓPEZ VÉLEZ, A.L. (en prensa). Las competencias de emprendimiento social, COEMS: Aproximación a través de programas de formación universitaria en Iberoamérica. REVESCO, Revista de Estudios Cooperativos.

SAMUELOWICZ, K., y BAIN, J. D. (2001). Revisiting Academics' Beliefs about Teaching and Learning. Higher Education, 41(3), 299-325.

TARDIF, J. (2006). L'évaluation des compétences. (Tremblay, L.). Québec: Les éditions de la Chenelière.

TIGELAAR, D., DOLMANS, D. H. J. M., WOLFHAGEN, I. H. A. P., y van der Vleuten, C. P. M. (2004). The development and validation of a framework for teaching competencies in higher education. Higher Education, 48(2), 253-268. doi:10.1023/B:HIGH.0000034318.74275.e4

VILLA, A., CAMPO, L., ARRANZ, S., VILLA, O., y GARCÍA, A. (2013). Valoración del profesorado de magisterio sobre el aprendizaje basado en competencias implantado. Profesorado. Revista de Currículum y Formación de Profesorado., 17(3), 35-55.

VILLA, A., y POBLETE, M. (2007). Aprendizaje basado en competencias. Una propuesta para la evaluación de las competencias genéricas. Bilbao: Mensajero. 
WILIAM, D. (2011). What is assessment for learning? Studies in Educational Evaluation, 37(1), 3-14. doi:10.1016/j.stueduc.2011.03.001

ZEGERS, F. E. (1991). Coefficients for interrater agreement. Applied Psychological Measurement, 15(4), 321-333.

ZWIERS, M., y MORRISSETTE, P. J. (1999). Effective Interviewing of Children: A Comprehensive Guide for Counselors and Human Service Workers. Ann Arbor, United States: Taylor y Francis.

\section{Correspondencia con los autores}

Manuel POBLETE RUIZ

Universidad de Deusto

Avenida de las Universidades 24

48007 Bilbao

e-mail: manuel.poblete@deusto.es

Donna FERNÁNDEZ NOGUEIRA

Universidad de Deusto

Avenida de las Universidades 24

48007 Bilbao

e-mail: donna.fernandez@deusto.es

Lucía CAMPO CARRASCO

Universidad de Deusto

Avenida de las Universidades 24

48007 Bilbao

e-mail: 1.campo@deusto.es

Marcelo NÖEL LÓPEZ

Universidad de Deusto

Avenida de las Universidades 24

48007 Bilbao

e-mail: marcelo.noel@gmail.com 


\section{ANEXO 1}

Resultados del Coeficiente de Gower en los 7 indicadores de las cuatro competencias evaluadas

\begin{tabular}{|l|c|c|c|c|c|}
\hline & Evaluador 1 & Evaluador 2 & Evaluador 3 & Evaluador 4 & Evaluador 5 \\
\hline Evaluador 1 & & 0,63 & 0,62 & 0,61 & 0,69 \\
\hline Evaluador 2 & & & 0,96 & 0,95 & 0,85 \\
\hline Evaluador 3 & & & 0,99 & 0,88 \\
\hline Evaluador 4 & & & & 0,89 \\
\hline Evaluador 5 & & & & \\
\hline
\end{tabular}

Tabla 5. Resultados de Coeficiente de Gower para Indicador 1: Emplea la escucha (Competencia Comunicación).

\begin{tabular}{|l|c|c|c|c|c|}
\hline & Evaluador 1 & Evaluador 2 & Evaluador 3 & Evaluador 4 & Evaluador 5 \\
\hline Evaluador 1 & & 0,69 & 0,81 & 0,87 & 0,73 \\
\hline Evaluador 2 & & & 0,79 & 0,77 & 0,70 \\
\hline Evaluador 3 & & & 0,89 & 0,89 \\
\hline Evaluador 4 & & & & 0,83 \\
\hline Evaluador 5 & & & & & \\
\hline
\end{tabular}

Tabla 6. Resultados de Coeficiente de Gower para Indicador 2: Escucha y entiende las ideas de los demás aunque sean opuestas a las suyas (Competencia Comunicación).

\begin{tabular}{|l|c|c|c|c|c|}
\hline & Evaluador 1 & Evaluador 2 & Evaluador 3 & Evaluador 4 & Evaluador 5 \\
\hline Evaluador 1 & & 0,86 & 0,79 & 0,81 & 0,81 \\
\hline Evaluador 2 & & & 0,85 & 0,78 & 0,88 \\
\hline Evaluador 3 & & & 0,88 & 0,88 \\
\hline Evaluador 4 & & & & 0,78 \\
\hline Evaluador 5 & & & & \\
\hline
\end{tabular}

Tabla 7. Resultados de Coeficiente de Gower para Indicador 3: Incorpora el sentido ético en otros ámbitos y se orienta en torno a él (Competencia Comportamiento ético). 


\begin{tabular}{|l|c|c|c|c|c|}
\hline & Evaluador 1 & Evaluador 2 & Evaluador 3 & Evaluador 4 & Evaluador 5 \\
\hline Evaluador 1 & & 0,79 & 0,82 & 0,70 & 0,81 \\
\hline Evaluador 2 & & & 0,87 & 0,75 & 0,88 \\
\hline Evaluador 3 & & & 0,88 & 0,89 \\
\hline Evaluador 4 & & & & 0,77 \\
\hline Evaluador 5 & & & & \\
\hline
\end{tabular}

Tabla 8. Resultados de Coeficiente de Gower para Indicador 4: Mantiene coherencia entre lo que piensa y lo que hace (Competencia Comportamiento ético).

\begin{tabular}{|l|c|c|c|c|c|}
\hline & Evaluador 1 & Evaluador 2 & Evaluador 3 & Evaluador 4 & Evaluador 5 \\
\hline Evaluador 1 & & 0,85 & 0,85 & 0,76 & 0,89 \\
\hline Evaluador 2 & & & 0,85 & 0,79 & 0,91 \\
\hline Evaluador 3 & & & 0,91 & 0,91 \\
\hline Evaluador 4 & & & & 0,85 \\
\hline Evaluador 5 & & & & \\
\hline
\end{tabular}

Tabla 9. Resultados de Coeficiente de Gower para Indicador 5: Realiza las tareas que le son asignadas dentro del grupo en los plazos requeridos (Trabajo en equipo).

\begin{tabular}{|l|c|c|c|c|c|}
\hline & Evaluador 1 & Evaluador 2 & Evaluador 3 & Evaluador 4 & Evaluador 5 \\
\hline Evaluador 1 & & 0,82 & 0,89 & 0,77 & 0,91 \\
\hline Evaluador 2 & & & 0,93 & 0,80 & 0,91 \\
\hline Evaluador 3 & & & 0,88 & 0,98 \\
\hline Evaluador 4 & & & & 0,86 \\
\hline Evaluador 5 & & & & \\
\hline
\end{tabular}

Tabla 10. Resultados de Coeficiente de Gower para Indicador 6: Se interesa por la importancia social de la actividad que se desarrolla en el grupo (Competencias Trabajo en equipo).

\begin{tabular}{|l|c|c|c|c|c|}
\hline & Evaluador 1 & Evaluador 2 & Evaluador 3 & Evaluador 4 & Evaluador 5 \\
\hline Evaluador 1 & & 0,81 & 0,90 & 0,84 & 0,90 \\
\hline Evaluador 2 & & 0,88 & 0,81 & 0,86 \\
\hline Evaluador 3 & & & 0,91 & 0,96 \\
\hline Evaluador 4 & & & & 0,93 \\
\hline Evaluador 5 & & & & \\
\hline
\end{tabular}

Tabla 11. Resultados de Coeficiente de Gower para Indicador 7: Anima al equipo a la consecución de objetivos (Competencia Liderazgo). 